\title{
An Innovative Technique to Maintain a Clear Surgical Field During Venous Anastomosis in Free Flap Transfer for Head and Neck Reconstruction
}

\author{
Pradipkumar Atodaria, MS, MCh", ${ }^{1 *}$; Viralkumar Patel, MS, DNB'; Kuldeepsinh Atodaria, MBBS3; \\ Nikunj Vithalani, MS, DNB'; Mishal Shah, MS, DNB ${ }^{4}$
}

Department of Plastic Surgery, Bharat Cancer Hospital \& Research Institute, Surat, Gujarat, India

${ }^{2}$ Surat Municipal Institute of Medical Education \& Research, Surat, Gujarat, India

${ }^{3}$ Government Medical College, Surat, Gujarat, India

${ }^{4}$ Department of Onco-Surgery, Bharat Cancer Hospital \& Research Institute, Surat, Gujarat, India

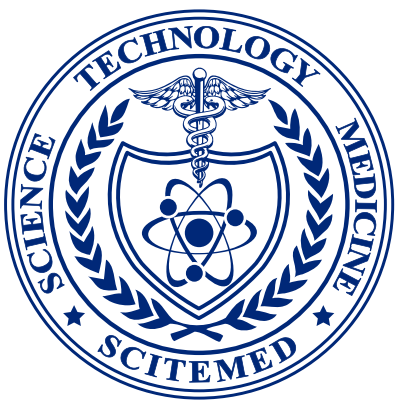

\begin{abstract}
Reconstruction of defects with microvascular free flaps is an important procedure after cancer resection. In the absence of the specific indications for granting priority to arterial or venous repair, we prefer to repair artery first. In doing so, however, a clear surgical field may not be obtained at the time of repair of first vena comitans because the second vena comitans may continue to bleed. The methods used to solve this problem may be clamping the second vena comitans or diverting the flow of the second vena comitans. In our practice, we used to divert the flow out of the surgical field rather than clamping the second vena comitans in order to reduce the risk of venous congestion of the flap. In this article, we describe a new developed technique for placing the second vena comitans into a cut glove finger to divert the bleeding of the freely draining vena comitans away from the venous anastomosis site.
\end{abstract}

\section{INTRODUCTION}

In micro-vascular surgery, there is no consensus regarding which vessel to be repaired first - the artery or the vein. For practical reasons, the deeper or more difficult to reach vessel is repaired first [1]. Repair of the artery first has the advantage of early flap revascularization and reducing the warm ischemia time. Arterial repair first may reveal the dominant venous drainage and aid in the selection of donor vein, if only one vein is to be repaired (depending upon the availability of recipient vein/s). The disadvantage of repairing the artery first is that, if the artery is unclamped after the anastomosis, bleeding from the second vena comitans makes the repair of vein difficult. If the second vein is also clamped, then venous congestion develops, which is well known to be detrimental to flap survival [2]. Alternatively, if the venous anastomosis is performed first, it delays revascularization of the flap.

We routinely perform the arterial anastomosis first and unclamp the artery during venous anastomosis. The aim of our innovative idea is to divert the bleeding from the freely draining vena comitans away from the venous anastomosis site. Therefore, we tried an innovative technique using the finger of a surgical glove to divert venous blood from the second vena comitans away from the surgical field.

\section{METHODS}

In oncological head \& neck reconstruction, we routinely use the free radial artery forearm flap, medial sural artery perforator flap, anterolateral thigh flap, and fibula osteocutaneous flap. In all these flaps, our innovative technique is used as and when needed. We always perform the arterial anastomosis first and unclamp the artery during venous anastomosis; however, we do not clamp the second vena comitans. We introduce the second vena comitans into one end of a cut glove finger rinsed with heparinised saline [5000 I.U. of heparin in $500 \mathrm{ml}$ saline] to divert the bleeding from the operative field. This technique is demonstrated in the following video: https://youtu.be/BhOiPG9WtpM

\section{DISCUSSION}

In an experimental study in an animal model, Thomson et al. [2] showed that flap failure was highest if the arterial anastomosis was performed first and immediately unclamped. This result was due to venous congestion as the vein was clamped during anastomosis [2]. In the study conducted by Thomson et al. [2], the average anastomosis time for artery and vein was $25 \pm 4$ minutes and $26 \pm 3$ minutes, respectively. The time taken for anastomosis is comparable to the actual average time taken during the surgery by us. However, the other experimental animal study by Zhang et al. [3] concluded that a brief venous stasis during anastomosis after the establishment of arterial inflow was not detrimental to flap survival and the sequence of anastomosis would not affect the outcome in both skin and muscle flap [3]. In this study by Zhang et al, the average anastomosis time for artery and vein was 8 to 10 minutes and 12 to 16 minutes, respectively. These timings are too fast compared to the average time taken during surgery in operation theatre. The difference in outcome/failure rate in the above two studies may be due to the total time taken for artery and vein.

If the time of anastomosis increases, injury to vessels due to clamp application becomes significantly higher [2]. Often, untoward circumstances arising during surgery may prolong the total anastomosis time even further.

At the Chang Gung Memorial Hospital where more than 800 flaps are performed per year, the usual practice is to repair the artery first and leave it unclamped while the vein is being repaired. Clamping of the vein has not caused any major problem in their practice [1]. We also routinely perform the arterial anastomosis first and unclamp the artery during venous anastomosis. If the second vena comitans is present, we reduce venous congestion by allowing the second vein to drain freely. Blood from the freely draining vena comitans will accumulate at the anastomosis site (Figure 1A). To avoid this unwanted accumulation of blood at the venous anastomosis site, we tried an innovative technique by introducing the second vena comitans into one end of a cut glove finger [rinsed with heparinized normal saline] to drain the blood away from the anastomosis site (Figure 1B, 1C). 

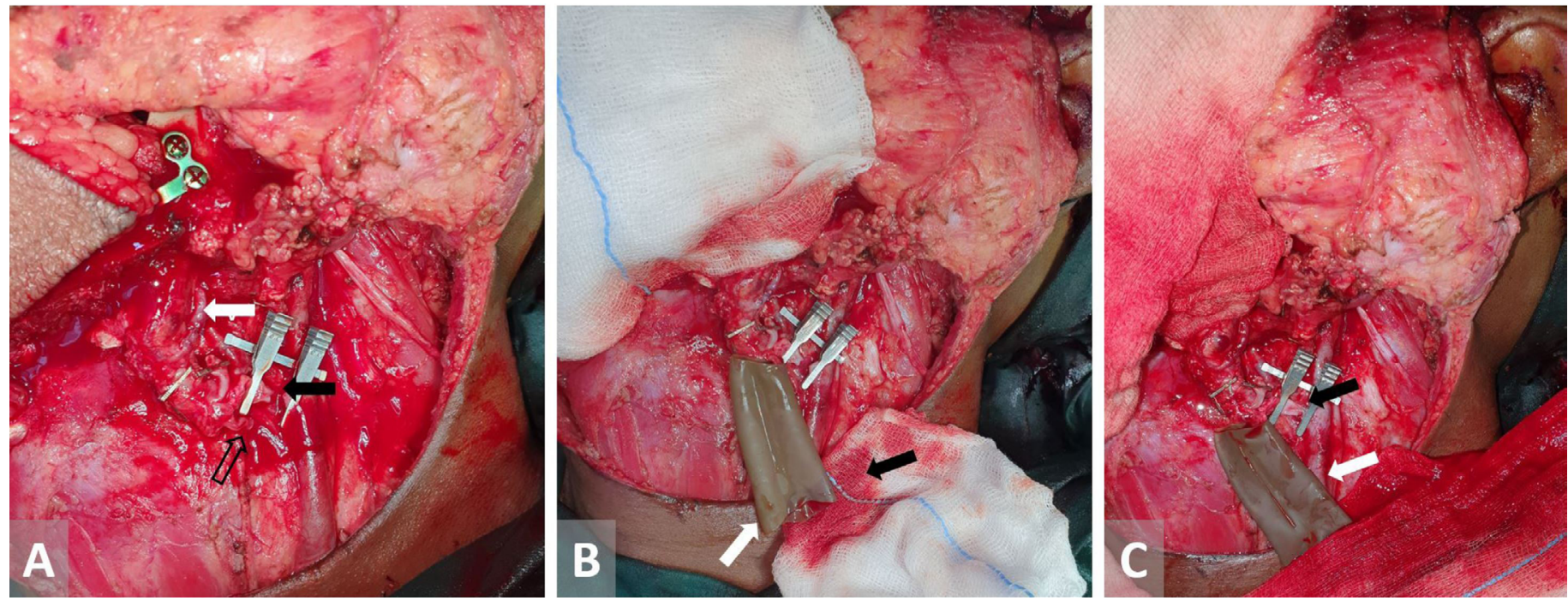

Figure 1. (A) The white arrow indicates peroneal artery anastomosis with left facial artery, the solid black arrow indicates vena commitans to be anastomose with tributary of internal jugular vein, and the hollow black arrow indicates un-clamped vena commitans bleed to anastomosis site.(B) The white arrow indicates unclamped vena commitans introduced inside finger glove, and the black arrow indicates gauze piece soiled with blood keeping anastomosis site clean. (C) The black arrow indicates vena commitans anastomosed with tributary of internal jugular vein in clear field, and the white arrow indicates unclamped vena commitans introduced inside finger glove draining over mop.

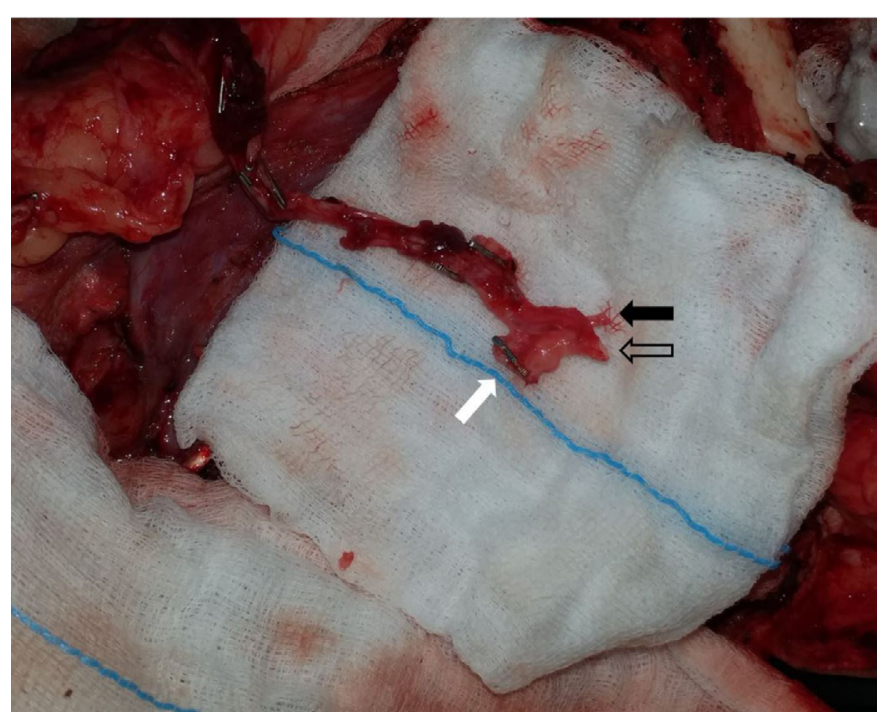

Figure 2. The solid black arrow indicates artery, the hollow black arrow indicates vein, and the white arrow indicates ligaclip applied over proximal tributary.

Sometimes (in anterolateral thigh and medial sural artery perforator flaps), the vena comitans join together to form a single larger vein which is easy to repair, and we dissect untill anastomosable length of larger vein is achieved. In such circumstances, we usually dissect and keep a proximal branch of the vascular bundle for about a centimeter before the application of ligaclip. After arterial repair, we remove the ligaclip from the vein to allow this proximal tributary of the vein to drain freely in cut finger glove to reduce venous congestion (Figure 2).

We had measured blood loss from the unclamped vena comitans by weighing mops before and after use. The average blood loss was $33 \mathrm{ml}, 21 \mathrm{ml}$, $14 \mathrm{ml}$, and $8 \mathrm{ml}$ in fibula osteocutaneous flap, anterolateral thigh flap, radial artery forearm flap, and medial sural artery perforator flap, respectively.

\section{CONCLUSION}

This simple innovative technique helps in achieving a clear surgical field at the time of venous repair by diverting the venous bleeding through a glove finger and at the same time preventing venous congestion of the flap.

\section{ARTICLE INFORMATION}

*Correspondence: Pradipkumar Atodaria, MS, MCh. Department of Plastic Surgery, Bharat Cancer Hospital \& Research Institute, Surat-Bardoli road, Saroli, Surat, Gujarat, 395010, India. Email: dratodaria@gmail.com

Received:Jul. 27, 2019; Accepted: Apr. 08, 2020; Published:Jun. 10, 2020

DOI: $10.24983 /$ scitemed.imj.2020.00128

Ethics Approval and Consent to Participate: The study is in accordance with the ethical standards of the 1964 Helsinki declaration and its later amendments or comparable ethical standards.

Funding: The study did not receive any specific grant from funding agencies in the public, commercial, or not-for-profit sectors.

Conflict of Interest: The authors report no financial or other conflict of interest relevant to this article, which is the intellectual property of the authors.

Copyright $\odot 2020$ The Authors. This is an open-access article distributed under the terms of the Creative Commons Attribution 4.0 International License (CC-BY).

\section{REFERENCES}

1. Wei FC, Tay SKL. Chapter 26: Principles and techniques of microvascular surgery. In: Neligan PC ,Gurtner, GC (Eds.). Plastic Surgery Principles in Plastic Surgery, vol. 1, 3rd ed. Elsevier; 2013.

2. Thomson JG, Kim JH, Syed SA, Reid MA, Madsen J, Restifo RJ. The effect of prolonged clamping and vascular stasis on the patency of arterial and venous microanastomoses. Ann Plast Surg 1998;40(4):436-441.

3. Zhang $F$, Pang $Y$, Buntic $R$, et al. Effect of sequence, timing of vascular anastomosis, and clamp removal on survival of microsurgical flaps.J Reconstr Microsurg 2002;18(8):697-702. 